\title{
NEUTRINOS FROM SN1987a IN THE IMB DETECTOR
}

\author{
Todd HAINES \\ Department of Physics, University of Maryland, College Park, MD 20742, USA
}

IMB Collaboration

\author{
C.B. BRATTON, D. CASPER, A. CIOCIO, R. CLAUS, M. CROUCH, S.T. DYE, S. ERREDE, \\ W. GAJEWSKI, M. GOLDHABER, T.J. HAINES, T.W. JONES, D. KIELCZEWSKA, W.R. KROPP, \\ J.G. LEARNED, J.M. LOSECCO, J. MATTHEWS, R. MILLER, M.S. MUDAN, L.R. PRICE, \\ F. REINES, J. SCHULTZ, S. SEIDEL, E. SHUMARD, D. SINCLAIR, H.W. SOBEL, L.R. SULAK, \\ R. SVOBODA, G. THORNTON and J.C. VAN DER VELDE
}

The University of California, Irvine, CA, USA

The University of Michigan, Ann Arbor, MI, USA

Brookhaven National Laboratory, Upton, NY, USA

Cleveland State University, Cleveland, OH, USA

The University of Hawaii, Honolulu, HI, USA

University College, London, UK

Warsaw University, Warsaw, Poland

Case Western Reserve University, Cleveland, $\mathrm{OH}, \mathrm{USA}$

The University of Illinois, Urbana, IL, USA

The University of Notre Dame, Notre Dame, IN, USA

Boston University, Boston, MA, USA

$A T \& T$ Bell Laboratories, Summit, NJ, USA

The University of Maryland, College Park, MD, USA

\begin{abstract}
A burst of eight neutrino events preceding the optical detection of the supernova in the Large Magellanic Cloud has been observed by the IMB detector. The events span an interval of $6 \mathrm{~s}$ and have visible energies in the range 20-40 MeV. Some comparisons between the signal and that expected from a supernova are made. We have found no other comparable burst during the operation of IMB.
\end{abstract}

According to conventional supernova theory [1], the recently observed supernova explosion SN1987a [2] in the Large Magellanic Cloud (LMC) should have released approximately $3 \times 10^{53} \mathrm{erg}$ of gravitational binding energy in a burst of $10^{58}$ neutrinos in a time interval of a few seconds. We report results from the observation of a burst of neutrino events, completely contained within the volume of the IMB water-Cherenkov detector, occurring close to the estimated time of the supernova collapse. The observed signal consists of eight neutrinos with energies in the range of $20-40 \mathrm{MeV}$, spaced over an interval of $6 \mathrm{~s}$. The background rate of contained events from cosmic ray produced neutrinos interacting in the detector is $-2 \mathrm{~d}$ in the range $20-2000$ $\mathrm{MeV}$. In this paper, we will briefly discuss the current status of the analysis of the burst as well as the angular distribution of the observed events [3].

The IMB detector [4], which was designed to search for proton decay, is located in the Morton-Thiokol salt mine near Fairport, Ohio $(41.7 \mathrm{~N}, 81.3 \mathrm{~W})$ at a depth of 1570 mwe (meter water equivalent). It consists of a rectangular tank $\left(22.5 \times 17 \times 18 \mathrm{~m}^{3}\right)$ filled with purified water. We consider the active volume for this search to be the entire detector, or about 6800 metric tons, somewhat greater than the nominal fiducial volume of 3300 metric tons used in the proton decay search. The six sides are instrumented with 2048 eight-inch photomultiplier tubes (PMTs) arranged on an approximate $1 \mathrm{~m}$ grid. The tubes are mounted on wave-shifter plates, which increases their light collection efficiency by a factor of about two. Each PMT provides timing and pulse-height information from which the vertex, direction, and energy of a charged particle track may be reconstructed.

For a seven hour period of time containing the observed burst, one-quarter of the PMTs, representing 

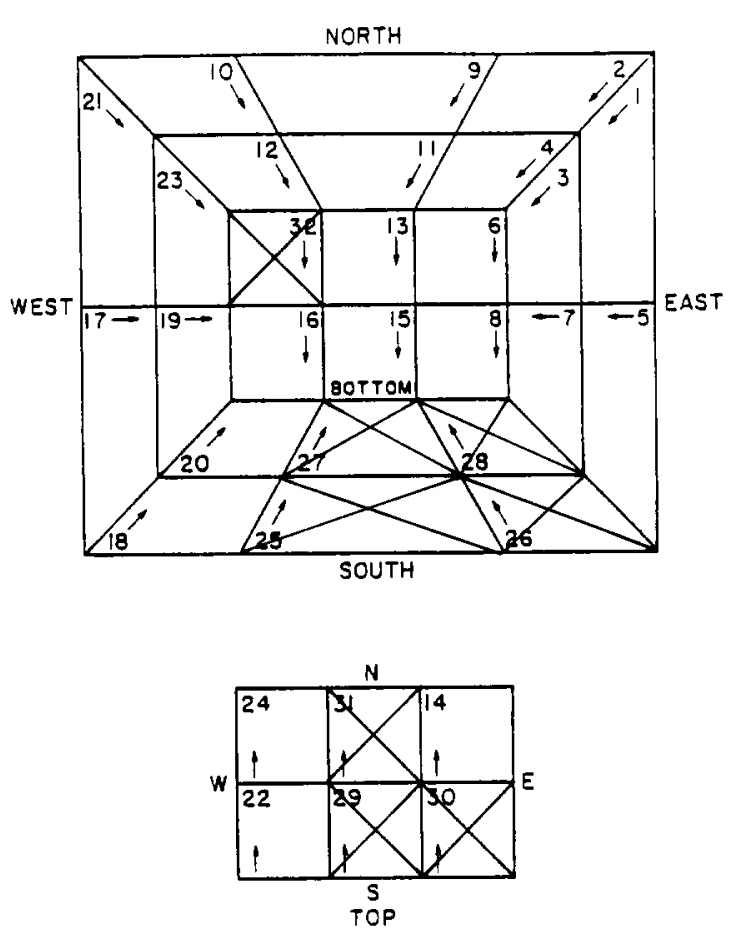

Fig. 1. A schematic view of IMB showing the division into patches. The inoperative patches have been crossed out.

essentially one contiguous region of the detector's walls, were not operational due to the failure of one of four power supplies. The detector is divided into 32 "patches", as shown in fig. 1, each patch being a local $8 \times 8$ array of PMTs. The inoperative power supply controlled the PMTs in patches 25-32 which, therefore, limited our sensitivity on much of the south and top walls. The local position of the supernova, at the time of the burst, was $42^{\circ}$ below the horizon and $28^{\circ}$ west of south.

The detector is triggered when about 20-25 PMTs fire in $\sim 50 \mathrm{~ns}$; this corresponds to about 40 or more PMTs firing in the 511 ns trigger recording window. The trigger efficiency as a function of visible energy has been determined by implementing the trigger condition on a sample of Monte Carlo electrons in the detector and is given in fig. 2 (see ref. [5]). These calculations have been been checked by simulating Cherenkov tracks in the detector using an artificial light source.

The signal of a supernova is expected to be a burst of low-energy neutrinos occurring over a period of seconds. In the search for low-energy neutrino events, an upper limit of 100 PMTs firing was set as an off-line acceptance criterion. To search for such a burst of neutrinos, a $6.4 \mathrm{~h}$ period starting at UT February 23, 5 h $00 \mathrm{~mm} 00 \mathrm{~s}$ (when the PMTs were inoperative) was selected and divided into non-overlapping $10 \mathrm{~s}$ intervals; the number of events with fewer than 100 PMTs

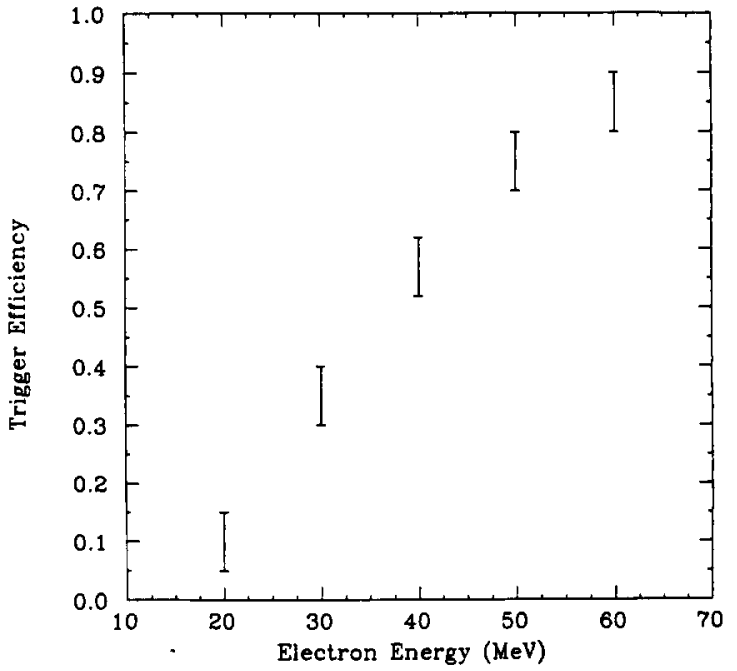

Fig. 2. Trigger efficiency as a function of electron energy averaged over an isotropic distribution in the full $6800 \mathrm{~m}^{3}$ volume of the partially operating detector. The error bars represent the systematic uncertainty in efficiency.

firing $(N)$ was determined for each interval. For $N \leq 5$, this distribution is fitted well by a Poison distribution with $\langle N\rangle=0.77$ events per interval. However, one interval is found to contain nine events.

Using the track imaging capabilities of this detector, the nine events during this time interval were studied in detail to determine if they could be due to charged particles entering the detector, or possibly spurious triggers of instrumental origin. One event was identified as a typical cosmic ray muon; the remaining eight events are identified as neutrino interactions in the detector volume. We estimate the efficiency for finding the neutrino-burst events, once the detector has triggered, is essentially $100 \%$ based on a double scanning technique. None of the events can be identified as a muon since no decay electron followed; we found no other neutrino events during this $6.4 \mathrm{~h}$ period.

The visible energies of these events were determined by correcting the observed PMT pulse heights for noise and geometric effects and comparing with detailed simulations of Cherenkov light production in the detector; these Monte Carlo programs have been crosschecked with samples of cosmic ray muons and muondecay electrons. If we assume these events are caused by electron antineutrinos interacting on free protons, then the visible energy corresponds closely to the neutrino energy. The uncertainties in these energy determinations are estimated to be $\pm 25 \%$ (statistical and systematic). Reconstruction of the individual track directions has an uncertainty of about $\pm 15^{\circ}$. The observed events, time of occurrence, number of PMTs firing, estimated energies, distance from the nearest PMT plane, and track 
Table 1

Characteristics of the contained neutrino burst events recorded on 23 February, 1987.

\begin{tabular}{|c|c|c|c|c|c|c|}
\hline \multicolumn{2}{|c|}{$\begin{array}{l}\text { Event }{ }^{\text {a) }} \\
\text { number }\end{array}$} & \multirow{2}{*}{$\begin{array}{l}\text { Time (UT) } \\
7: 35: 41.37\end{array}$} & \multirow{2}{*}{$\begin{array}{l}\text { Number } \\
\text { of PMTs }\end{array}$} & \multirow{2}{*}{$\begin{array}{l}\begin{array}{l}\left.\text { Energy }{ }^{\mathrm{b}}\right) \\
(\mathrm{MeV})\end{array} \\
38\end{array}$} & \multirow{2}{*}{$\begin{array}{l}\begin{array}{l}\text { Angular Distribution } \\
\text { (deg) }\end{array} \\
74\end{array}$} & \multirow{2}{*}{$\begin{array}{l}\begin{array}{l}\text { PMT Distribution d) } \\
\text { (m) }\end{array} \\
7.9\end{array}$} \\
\hline 1 & 33162 & & & & & \\
\hline 2 & 33164 & 41.79 & 61 & 37 & 52 & 3.2 \\
\hline 3 & 33167 & 42.02 & 49 & 40 & 56 & 3.5 \\
\hline 4 & 33168 & 42.52 & 60 & 35 & 63 & 4.1 \\
\hline 5 & 33170 & 42.94 & 52 & 29 & 40 & 1.3 \\
\hline 6 & 33173 & 44.06 & 61 & 37 & 52 & 4.8 \\
\hline 7 & 33179 & 46.38 & 44 & 20 & 39 & 5.9 \\
\hline 8 & 33184 & 46.96 & 45 & 24 & 102 & 5.0 \\
\hline
\end{tabular}

a) The event numbers are not sequential. Interspursed with the contained neutrino events are 15 entering cosmic ray muons.

b) Error in energy determination is $25 \%$ (systematic plus statistical).

c) Individual track reconstruction uncertainty is about $15^{\circ}$.

d) PMT distance is the distance of event vertex from the nearest PMT plane; vertex resolution is about $1 \mathrm{~m}$.

angles with respect to the supernova are given in table 1.

If we assume that the uncertainty in energy $( \pm 25 \%)$ for our events is a Gaussian distribution about the nominal energy then we can make a determination of the (assumed) electron antineutrino spectrum from the supernova. We note these assumptions may not be valid; the validity of these assumptions is under investigation. We take a unit normalized Gaussian distribution centered at the nominal energy $E$ with $\sigma=0.25 E$ to represent the probability that the event actually had energy $E$. If we further assume that the events are due to inverse beta decay on free protons (see below for a discussion) we can estimate the incident neutrino flux. Folding our energy dependent detection efficiency, the energy dependent neutrino cross section, and the number of free protons in the detector together with the observed energy distribution then gives the antineutrino spectrum [6]. A fit to the spectrum with an exponential distribution then gives a total antineutrino flux of $8 \times$ $10^{8} \mathrm{~m}^{-2}$ at the detector. The total antineutrino output of the supernova is the $3 \times 10^{56}$ corresponding to a luminosity of electron antineutrinos of $1 \times 10^{52} \mathrm{erg}$ above $20 \mathrm{MeV}$, in reasonable agreement with expectations.

If these events are in fact due to inverse beta decay, then the resulting distribution of events should be relatively isotropic with respect to the initial neutrino direction. We have completed initial studies of the effect of the inoperative PMTs on the observed angular distribution of events in the detector. For example, using a sample of simulated $30 \mathrm{MeV}$ electrons distributed isotropically in the detector on which the trigger criterion is applied ("turning off" the inoperative PMTs), we can study the angular bias. If we let the direction from the LMC define the $z$-axis of a spherical coordinate system, fig. 3shows the azimuthal and polar distributions expected from an isotropic distribution of 20 and $30 \mathrm{MeV}$ events with respect to the LMC direction. The resulting forward asymmetry is $(2 \pm 4) \%$, a negligible effect. This result is true only when the efficiency is averaged over
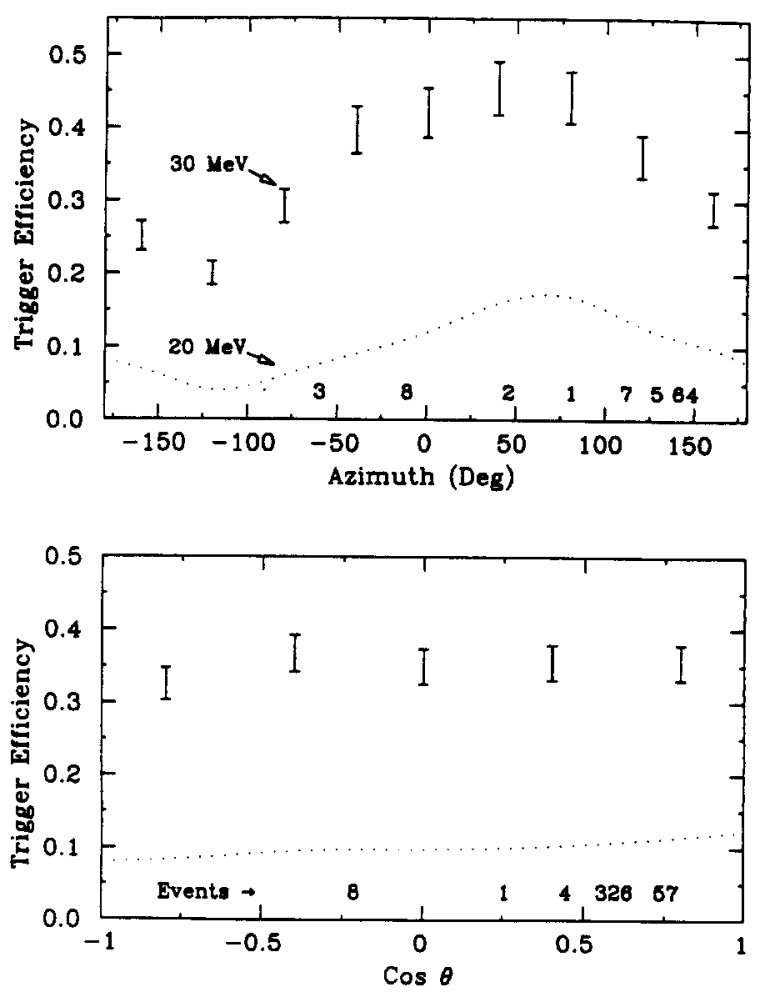

Fig. 3. Angular dependence of the trigger efficiency for 20 $\mathrm{MeV}$ (dots) and $30 \mathrm{MeV}$ electrons. Angles are measured with respect to the direction away from the supernova. The error bars represent the uncertainty in angular dependence; an additional overall systematic uncertainty applies (see fig. 2). The angles of the eight events listed in table 1 are indicated by numerals. 
the entire detector; specific subvolumes of the detector do show sizable polar angle biases. If we consider only the positions of the events in the detector, there is not a significant polar angle bias.

The polar angles of the eight observed events are also shown in fig. 3. The observed distribution appears peaked in the forward direction with the angles of 7 of the 8 events between $39^{\circ}\left( \pm 15^{\circ}\right)$ and $75^{\circ}\left( \pm 15^{\circ}\right)$. This does not seem to be a good fit to either neutrino-electron elastic scattering, which is strongly forward peaked with $\theta \leq 45^{\circ}$ (including multiple scattering and reconstruction errors), nor to the nearly isotropic distribution expected from inverse- $\beta$ decay. We have not yet evaluated the precise likelihood for either of these interpretations. We note, however, that the 4 events observed by Kamiokande [7] above the IMB threshold all have polar angles in a similar range of $18^{\circ}\left( \pm 18^{\circ}\right)$ to $38^{\circ}\left( \pm 22^{\circ}\right)$.

One can crudely estimate the likelihood of an isotropic distribution by simply assuming a probability of $50 \%$ that an event will lie in the forward hemisphere. This estimation is crude because it ignores the smearing caused by multiple scattering and reconstruction; therefore, this will result in an underestimate of the actual likelihood but it should at least be illustrative. The probability that 7 out of the 8 events will lie in the forward hemisphere is then about 3\%. Initial estimates of this probability that include the smearing effects mentioned above yield a likelihood of about $10 \%$. Obviously, if one includes some or all of the Kamiokande events that are above the IMB threshold, this probability drops drastically.

Using the experience gained with the neutrino burst from SN1987a as a guide, we can now search for evidence of stellar core collapses which were not detected optically; the lack of an optical sighting could be because it was absent (the stellar envelope was not ejected), small, or obscured. The IMB experiment has been in operation since September 1982. We have completed initial searches [8] for similar neutrino bursts throughout all of our data. This includes 335 live days of data before the detector was upgraded (September 1982-July 1984) and 140 live days after the final upgrade [9] (April 1986-February 1987) until the time of the supernova. We have found no other comparable bursts of neutrinos and can therefore set an upper limit on the rate of stellar core collapse in our Galaxy of $<1.8 /$ yr at $90 \% \mathrm{CL}$.

\section{Acknowledgements}

This work was supported in part by the U.S. Department of Energy. The hospitality of the Morton-Thiokol company, in whose Fairport mine these events were recorded, is greatly appreciated. One of us (T.H.) thanks the MP-Division at LAMPF for their hospitality.

\section{References}

[1] S.A. Colgate and R.H. White, Astrophys. J. 143 (1966) 626; J.R. Wilson et al., Ann. N.Y. Acad. Sci. 470 (1986) 267;

J.N. Bachall, A. Dar and T. Piran, Neutrinos from the Supernova in the LMC, Inst. for Avd. Study preprint (Feb. 1987);

T. Walker and D. Schramm, Resonant Neutrino Oscillations and the neutrino Signature of Supernova, FermilabPub-86/133A (Sept. 1986);

S.A. Bludman and P.J. Schnider, Theoretical Significance of Detecting Neutrinos from Supernova 1987A, University of Penn. UPR 0326T (March 1987).

[2] W. Kunkel and B. Madore, IAU Circular 4316 (Feb. 24, 1987).

[3] For further details concerning the neutrino burst please see: R.M. Bionta et al., Phys. Rev. Lett. 58 (1987) 1449.

T.J. Haines et al., Proc. of the Telemark IV Workshop on Neutrino Masses and Astrophysics (UCI Neutrino No. 87-15) Ashland, WI (March 1987)

[4] Further details concerning the IMB detector can be found in: R.M. Bionta et al., Phys. Rev. Lett. 51 (1983) 27;

B.G. Cortez et al., Phys. Rev. Lett. 52 (1984) 1092;

T.J. Haines et al., Phys. Rev. Lett. 57 (1986) 1986;

R. Claus et al., submitted to Nucl. Instr. and Meth. and references therein.

[5] Figs. 2 and 3 and table 1 represent our best estimates of the detection efficiency, energy, and directions for these events at the time of writing this paper. Please see C.B. Bratton et al., in preparation, for our final determination of these quantities.

[6] We have used the value of $7.5 \times 10^{-44}(E /(1 \mathrm{MeV}))^{2} \mathrm{~cm}^{2}$ for the inverse beta decay cross section. There are a total of $3.3 \times 10^{33}$ free protons in the detector.

[7] K. Hirata et al., Phys. Rev. Lett. 58 (1987) 1490.

[8] For more details please see the report of S.T. Dye for the IMB Collaboration at the 20th Int. Cosmic Ray Conf., Moskow (August, 1987).

[9] Please see T.J. Haines et al., Proc. of the 6th Workshop on Grand Unification (UCI Neutrino No. 150, July, 1985) eds., S. Rudaz and T.W. Walsh, Minneapolis (1985) for a discussion of the upgrades. 